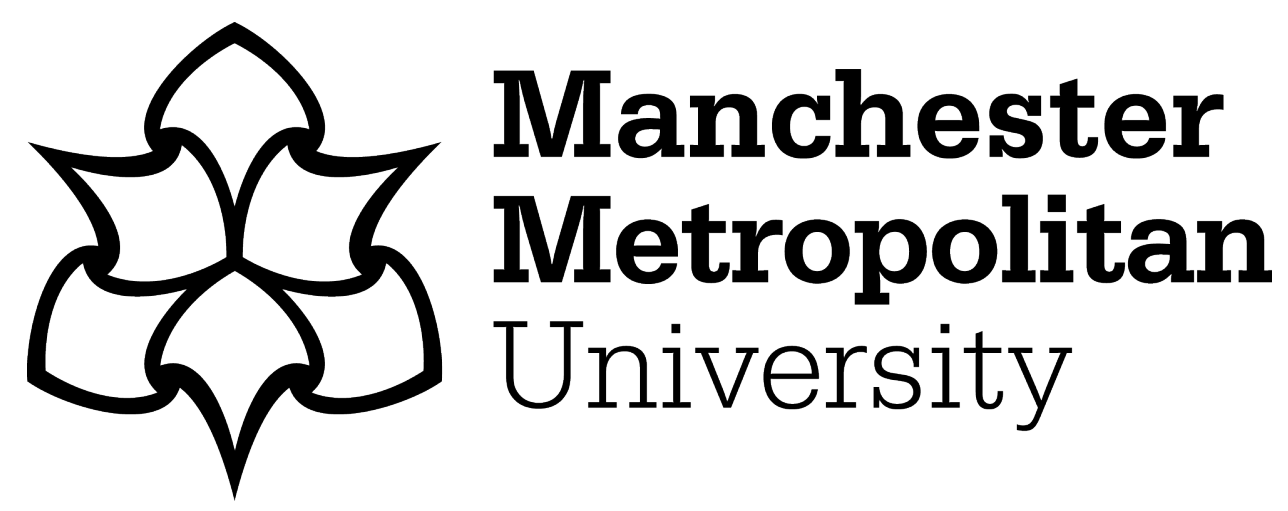

Hutchinson, Anthony and Moerman, Daniel E (2018) The Meaning Response, 'Placebo' and Methods. Perspectives in Biology and Medicine, 61 (3). pp. 361-378. ISSN 0031-5982

Downloaded from: https://e-space.mmu.ac.uk/621529/

Version: Accepted Version

Publisher: Johns Hopkins University Press

Please cite the published version 


\title{
The MeANing Response, "PlaceBo," AND METHODS
}

Phil hutchinson* AND DANIEl E. MOERMAN†

\begin{abstract}
In 2002, Dan Moerman outlined three candidate explanations for the "placebo response": the "conditioned stimulus-response," Irving Kirsch's "response-expectancy" explanation, and the "meaning response." The meaning response, Moerman argued, was the only one of the three candidate explanations that could cover all the data, gained from decades of RCTs and centuries of historical record. Moerman went so far as to propose replacing the term "placebo effect/response" with the term "meaning response," because people are not responding to placebos, since there is nothing to respond to; people are responding to meanings. There is evidence of medically significant meaning responses where there is no evidence for conditioning. Similarly, there is evidence for such responses where those subject to them lack the knowledge - epistemic capital — required to form the beliefs which might constitute an expectation. Something else, neither conditioning nor propositional attitudes, explained placebo responses, and Moerman proposed the meaning response. While the authors consider the meaning response to avoid the pitfalls of conditioning and response-expectancy, it has been subject to criticism. The criticisms have focused on what is seen as the explanation falling foul of the naturalistic demand and not fitting
\end{abstract}

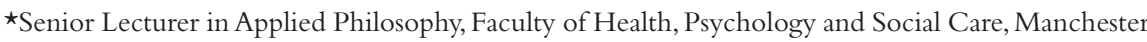
Metropolitan University.

†William E. Stirton Professor Emeritus of Anthropology, University of Michigan-Dearborn, and Research Associate, Botanical Research Institute of Texas.

Correspondence: Phil Hutchinson, Senior Lecturer in Applied Philosophy, Faculty of Health, Psychology and Social Care, Manchester Metropolitan University, Brooks Building, 53 Bonsall Street, Manchester, M15 6GX, United Kingdom.

Email: p.hutchinson@mmu.ac.uk.
} 
with prevalent predilections in the philosophy of mind and cognitive science. This article seeks to allay these worries and proposes the inclusion of ethnomethodological fieldwork in future research.

\section{Is There A PHENOMENON?}

Is there a response, which is not accounted for by regression to the mean (statistical artefact), natural history (people recovering over time), the Hawthorne effect (subjects behaving a certain way because they are being observed)?

$\mathrm{T}$ HE TERM PLACEBO COMES TO US from the Latin for "I shall please," indicating that the phenomenon known as the "placebo effect" or "placebo response" has been familiar to medical practitioners for a number of centuries, at least. As we reached the mid-20th century and randomized controlled trials (RCTs) became a central feature of medical research, the use of controls and blinding in those trials allowed for the isolation of the placebo effect in ways that made it much clearer that there was a phenomenon here which had significance, not only for the practice of medicine but also for understanding more generally the ways in which human beings meet and respond to the world. Well-conducted and designed RCTs, which include both "no treatment" and placebo controls, allow researchers to establish that what had historically been referred to as the "placebo effect" or "placebo response" was indeed a discrete medical phenomenon, one that wasn't reducible to regression to the mean, nor explained away as patients misreporting their symptoms.

So, RCTs and their meta-analyses provided the data that afforded placebo researchers a kind of epistemic confidence and warrant for claiming the placebo effect to be a phenomenon worthy of scientific investigation. Nevertheless, this hasn't prevented some from continuing to be placebo-skeptics and questioning whether there is a discrete phenomenon here. The go-to work for the skeptics is that published by Asbjorn Hróbjartsson and Peter Gøtzsche (2001, 2010). They conducted a systematic review of placebo-controlled RCTs: RCTs comprised of a placebo control, a no-treatment control, and a group receiving treatment. What their analysis seemed to show was that on average placebos produced outcomes only a little better than the no treatment groups. Based on this average, the authors concluded that "placebo" was actually a mislabeled natural history effect: people often tend to recover naturally over time. Headaches go away over time, pain subsides, rashes tend to clear up, and so on: the natural history of many ailments is that they pass.

While Hróbjartsson and Gøtzsche's work is largely rejected by placebo researchers, it is included in the Cochrane Library and therefore exercises influence on wider discussion among medical professionals. However, as Jeremy Howick and colleagues at Oxford subjected Hróbjartsson and Gøtzsche's work to scrutiny, they found that the conclusions that had been drawn were misleading (Ho- 
wick 2016; Howick et al. 2013). When you apply the same methods of review to the same body of data, but this time comparing the "real" treatment group with the no-treatment group, you find that on average, across all the studies, the real treatment group fare little better than the no-treatment and the placebo groups. It transpired that the conclusions drawn by Hróbjartsson and Gøtzsche were artefacts of their rather crude method of analysis: the production of the mean based on averaging a diverse range of RCTs, where the diversity extended so far as the definitions of placebo, "no treatment," and "real treatment." Put another way, Hróbjartsson and Gøtzsche set about averaging a diverse range of RCTs without addressing and controlling for the diversity.

Let's see if we can pump our intuitions a little around this issue by illustrating the problem with a hypothetical example. Let's say we have a couple of statisticians (commissioned perhaps by accountants for a commercial airline) who want to find out if you've more chance of living if you fall out of an aircraft with a parachute on, than without. Let's call them Starel and Olliardy. Starel and Olliardy take a whole host of studies that document people exiting an aircraft while it is in the air, with a view to producing a systematic review of those studies. Taking the studies as a whole and looking at the group who had parachutes and those who didn't have parachutes, they count up the survivors from each group and divide that total by the total in each of the respective groups, thereby arriving at their mean average survival rate for the with and without parachute groups. Having done this, they compare the averages and find, to their surprise (and the gratitude of the accountants), that there is little benefit to wearing a parachute. What's happened?

Well Starel and Olliardy haven't compared like with like. Their systematic review contained some studies where the subjects were exiting the aircraft, with or without a parachute, into enemy territory in a war zone. They had others where the aircraft was at such a high altitude that those exiting the aircraft required breathing equipment or insulation if they were to have good chance of surviving, yet this wasn't controlled for in their review. They had other studies that skewed their mean because while it was known that the batch of parachutes were faulty, our analysts hadn't adjusted for this. To compound matters, Starel and Olliardy didn't differentiate between deaths where ground impact was the proximate cause and deaths where other factors, such as a hostile natural environment or the availability of medical attention, played a significant causal role in determining survival rates.

So what we wanted to know was whether parachutes save lives. But we didn't obtain an answer to that question, because our reviewers, Starel and Olliardy, failed to be precise in asking the question and thus in their analysis of the data. The point is that there are many factors that impinge on survival rates for those exiting aircraft in the air, and only some of these are related to wearing a (functioning) parachute. If your study does not factor in and control for these other 
factors, then it will tell you next to nothing useful about the life-saving benefits of parachute-wearing, other things being equal. This is the point of control design.

To return to Hróbjartsson and Gøtzsche, they made a similar error to that of our hypothetical systematic reviewers of parachutes, because just as some medical conditions are more susceptible to medical treatment than are others, so some medical conditions are more susceptible to placebo treatment than others. Similarly, while some medical interventions work better than others, some placebo interventions work better than others too. Why this is so is the subject of our next section. Just as Starel and Olliardy's work can be misinterpreted as showing that no parachute is basically as good as a parachute for those exiting aircraft at altitude, Hróbjartsson and Gøtzsche misinterpreted their own review as showing that no treatment at all (remaining on waiting lists) was almost as effective as the administering of a placebo. In the parachute case, our intuitions tell us something must have gone wrong, and on investigation we discover what it is. In the case of Hróbjartsson and Gøtzsche, intuitions are, perhaps, less likely to lead to scrutiny, though it transpired things had gone wrong. Here's how Howick (2016) summarized the problem:

By lumping very different studies together it made the average effect misleading. The average colour of apples and oranges is brown, but unless they are rotten, neither oranges nor apples are brown. Their review included treatments for the common cold, alcohol abuse, smoking, herpes, marital discord, Alzheimer's disease, "undiagnosed ailments," and many others. The placebos were equally heterogeneous. Besides sugar pills, the "placebos" in their study included relaxation (classified as a treatment in some of the studies and as a placebo in others), leisure reading, answering questions about hobbies, reading newspapers, favourite foods, talking about daily events, football, vacation activities, books, movies, and television shows. Even if the average placebo effect (for any placebo for any disease) is quite small, some placebos for some things could be quite effective.

This raises two issues which interest us here, for the purposes of the present paper. First, it is interesting to surmise why, in the case of Hróbjartsson and Gøtzsche's study, so many seemed so satisfied with the conclusion that they were invited to produce a review for the Cochrane Foundation, when the methods were quite straightforwardly flawed, as readily exposed by Howick and colleagues. We suggest that the answer points to the need for philosophical scrutiny. Hróbjartsson and Gøtzsche's study seems intuitive to many, because it seems to support background materialist assumptions. Put another way, if one's spontaneous philosophical commitments are unreflectively materialist, then Hróbjartsson and Gøtzsche's conclusions will fit with those commitments, and this might mitigate against subjecting those conclusions to critical scrutiny. However, when it is found that almost the same conclusion follows for the medical intervention when 
the same systematic review of the same data is conducted, then that initial intuition is challenged.

Second, Hróbjartsson and Gøtzsche's work on placebo also trades on a somewhat liberal employment of the term, which seems to encompass things that might equally, if not more, appropriately be defined as either treatment or no treatment. For example, as Howick notes (see above) relaxation is defined as "placebo" in some of the studies under review, but as "treatment" in some of the others. Suffice to say, for present purposes, that this is a significant problem. "Placebo studies" as a program of enquiry needs to be clear about the phenomenon under study.

We're reminded here of $\$ 308$ of Ludwig Wittgenstein’s Philosophical Investigations, in which he remarks to the effect that both behaviorism and cognitivism, as theories of mind, fail to give an adequate account of their use of the term process as they employ that term (Wittgenstein 2009). References to "mental process" remain systematically unclear, whether employed in attempts to build a theory of thought and action (cognitivism) or as part of claims to reject the explanatory worth and thereby eliminate "mental process" from explanations of thought and action (behaviorism). As Wittgenstein reminds us, whether you want to produce a theory of something in pursuit of explanation or you want to eliminate the same, you need to have a grasp of-you need to be able to provide an account of - the grammar for that which you are theorizing or eliminating.

\section{EXPLAining THE PHENOMENON}

What is "placebo"?

It seems, then, that we do have a phenomenon. That which has come to be known as the "placebo response" is a phenomenon worthy of investigation. Well-designed RCTs with appropriate controls demonstrate there is something there that cannot be explained away by reference to regression to the mean, natural history effects, patient reporting bias, and the Hawthorne effect. What is it? Is it part of a wider phenomenon to be explained, or is it a singular, discrete phenomenon? Well, let us begin with what we know: for a wide range of medical conditions, the administering of a sham intervention-a biochemically inert dummy pill, or a sham surgical intervention-elicits medically significant recovery or relief from symptoms, recovery or relief that cannot be explained away as instances of regression to the mean, natural history, or the Hawthorne effect. We know it is not something smuggled in, so it is something else. Furthermore, for many medical interventions, where a pharmacologically active or surgical intervention is made, we know that success rates are often only explainable by reference to more than the pharmacology or the surgery; put slightly differently, medical treatment cannot simply be reduced to biochemistry and surgery. 
The "placebo response" (a term we ultimately want to forgo) is the measurable, observable, or felt improvement in health or behavior not attributable to a medication or invasive treatment that has been administered. ${ }^{1}$ Phrased another way: "the pills were inert; but taking a pill wasn't inert, and the brand name wasn't inert" (Moerman 2002, 19). We might add that "and where pills are not inert but have proven pharmacological efficacy, that efficacy is often enhanced in medically significant ways by the meaning specific social practices of pill-taking and administration have for those involved, in addition to the meaning and symbolic status of the packaging design, brand name, and site of administration has for those taking the pill."

For now, we'll continue to use the term placebo, though we've already hinted that the phenomenon that is accessed via the observation of "placebo responses" goes beyond "placebos"; we'll say more about this below. Let us look instead for the moment at the candidate explanations of placebo found in the literature. That which has traditionally been referred to as the "placebo effect/response" tends to be explained by appeal to two explanations: first, a conditioned response, which therefore has the character of a mechanism, a little like a reflex response, only one that emerges from conditioning (Ader 1988); or second, an expectancy response, which is therefore characterized on the model of belief and expectation-belief that " $x$ " leads to expectation that "x," triggering response "y" (Kirsch 1985). We'll look at these in turn.

\section{Conditioned Stimulus-Response}

The locus classicus of the conditioned stimulus-response is Pavlov's dogs. Here the conditioning is said to be comprised of a number of stages: patterned causal sensory stimuli (a) [sound of bell ringing when food appears], that are reliably associated over time with another distinct set of sensory stimuli (b) [taste and scent of food, satisfaction of appetite], which results in the development of a "trigger mechanism." Once developed, this "mechanism" becomes operative in the presence of sensory impacts (a) [the ringing of the bell] but the absence of (b) [the food], leading to certain physiological responses hitherto associated with sensory stimuli (b), such as salivating, now being "triggered" solely by sensory stimulus/ stimuli (a).

This is, it seems to us, the dominant folk explanation of placebo responses, but it fails to stand up to scrutiny. As an explanation, its appeal can be traced to

\footnotetext{
${ }^{1}$ Some researchers have proposed differentiating between the terms "placebo response" and "placebo effect" (see, for example, Blease, Bishop, and Kaptchuk 2017), such that the former is employed to encompass responses that might well be explained away as Hawthorne effects, regression to the mean, and so on, while "placebo effects" picks out the distinctive phenomenon. We do not operate with this distinction because (1) we don't find it helpful; (2) it has not been widely adopted; (3) it doesn't seem to have any relationship to the current grammar of either term; and (4) it begs the question somewhat in a research area where the precise nature of the phenomenon is still a matter of disagreement.
} 
a prevalent spontaneous philosophy, which leads simple mechanistic "explanations" to gain currency. However, conditioning cannot explain placebo responses for three reasons, which become evident when surveying the data.

First, classical-Pavlovian — conditioning has been widely abandoned as a viable theory, owing to a wealth of arguments that show that there is no such thing as Pavlovian conditioning as traditionally conceived and as it has taken hold in the folk imagination; rather, there is the learning and making of associations (Rescorla 1988). Second, there is an absence of evidence for the existence of a conditioning stage in many documented cases of placebo responses. Even if there were such a thing as the conditioning of popular psychological mythology, the necessary conditions for such conditioning in many cases of placebo responses just are not present. And third, problems arise due to the role of cultural variance: studies in which experimental subjects would be said to have been subject to the same conditioning (where there appears to be "evidence" of conditioning) produced significantly different outcomes when conducted on different groups from different cultures (see Moerman 2000). Put a different way, conditioning cannot account for the cultural indexicality/variance widely observed in studies of placebo responses because cultural differences often track different responses in people who might otherwise be said to have experienced the same conditioning or training (Moerman 2002).

\section{Response-Expectancy}

In the 1980s, Irving Kirsch proposed the response-expectancy explanation for placebo responses. This putatively explains placebos as responses to expectations: for example, you expect to find it difficult to sleep having had a double espresso late at night, and your expectation is based on your beliefs that caffeine is a stimulant and that a double espresso gives you a strong dose of caffeine. Given this expectation, you do indeed suffer disrupted sleep. However, unbeknownst to you, your host last night had run out of your favored java blend and, in a bid to avoid the shame that would inevitably follow from such a social faux pas, elected to give you a Swiss water decaffeinated double espresso without informing you. So your poor sleep was a placebo response, caused by your expectation, which was based on your (false) belief that the coffee you consumed was caffeinated (Kirsch 1985, 1997; Kirsch and Weixel 1988). Here's how Kirsch put it in 1997:

Response expectancy is the anticipation of one's own automatic reactions to various situations and behaviors. For example, a person may expect to feel more alert after drinking a cup of coffee, to feel less pain after taking pain medication, to feel intoxicated after drinking alcoholic beverages, and so on. Expected outcomes of this sort are among the things we consider in choosing courses of action (e.g., deciding whether to drink a cup of coffee or take pain medication). 
While response-expectancy is probably the current dominant explanatory framework employed in placebo studies, we have serious misgivings.

Response-expectancy logically implies the presence of beliefs with propositional content, or to put it another way, it presupposes belief that. The problem here is that placebo effects have been demonstrated time and time again to operate where there is no evidence for such belief. This is not a point based simply on patients/responders being unable to articulate their belief that intervention $\mathrm{X}$ is effective, it is rather that studies have shown that those responding to a placebo often do not have the knowledge- the epistemic resources - required to form the belief as to the benefit of the relevant factor. Put more expansively, on the standard view of cognition, to expect something that is not present to one, one has to represent the putative state of affairs one is expecting. The problem is that in many documented cases of placebo responses there is simply no evidence that those responding to the sham intervention have such a propositional attitude (unlike in the coffee example). However, and this is the more telling point, there are many cases of placebo responses where those responding to sham treatments in placebo-type ways do not have the epistemic resources, or even access to them, that might enable them to form the required belief. It is not merely that there are cases of placebo responses where those who respond do not appear to have the beliefs that would be required in order to form the expectation, it is that they don't have access to the data required to serve as the building blocks for such an expectation.

Consider the following example: two Italian studies showed that when men and women are given blue or orange placebo sleeping tablets, the women fall asleep much more quickly than the men when given blue placebos, and the duration of sleep is longer for women given blue placebos than it is for men (Cattaneo, Lucchelli, and Filippucci 1970; Lucchelli, Cattaneo, and Zattoni 1978). According to these studies, it would seem that in Italy, in the late 1960s and 1970s, blue pills inhibited sleep in men, or promoted it in women, or both, at least compared to orange pills. In the absence of further anthropological and ethnographic data, one can do little more than surmise why this divergence in effects was evidenced in the trial. However, what is relevant to our discussion here is that there is no period in these experiments, or in Italian life, where people are trained to sleep (or not) with exposure to "unconditioned stimuli," by taking blue tablets laced with barbiturates (for women), or with dextroamphetamine (for men). Indeed, we'd go so far as to suggest that it is distinctly unlikely that Italian women, in general, have grounds for believing that blue is a soporific color, while their male counterparts have grounds for believing the opposite. The ways in which the world has meaning for us cannot be reduced to belief that; meaning can emerge from associations and connotations, in addition to denotations. The response-expectancy explanation is constrained by being tied too closely to belief that, and therefore to propositionality. 
How this might exercise constraint over research is illustrated by the discussion in a well-known, classic study of the placebo effects of branded over non-branded aspirin. Branthwaite and Cooper (1981) write:

In each group the standard 50-tablet canister of the analgesic was used. The outward appearances of the branded packs (groups B and D) were the same and were identical with those available over the counter in the United Kingdom. The brand tested is one of the most popular, non-soluble aspirin-based analgesics in the United Kingdom and has been widely available for many years and supported by extensive advertising. The unbranded, plain packs (groups A and C) were labelled "analgesic tablets" with the same instructions for use and tablet contents as the standard branded pack. The tablets in the branded packs were endorsed with the manufacturer's design and those in the unbranded packs were plain. The active ingredient in groups $\mathrm{C}$ and $\mathrm{D}$ was $325 \mathrm{mg}$ aspirin per tablet; the tablets in these two groups had the same formulation and in-vitro and in-vivo release characteristics. Placebo and active tablets were the same size, shape, colour, and weight, although they were not specifically matched for taste following normal practice in trials with non-soluble products. (1576)

What interests us in this analysis is the following: when the analysis of the trial data is undertaken in the article, not only does the specific brand go without discussion (perhaps the authors weren't allowed to name the brand), but so do the specifics of the branding. The authors do not discuss such things as the choice of brand name and its potential associations and connotations, nor do they discuss such things as the packaging design, color, and price, and the meaning that might emerge from that. Rather, in noting that the branding had effects on both previous users of the brand and non-users, they surmise that this might emerge from the brand's general reputation. Unfortunately, we are not told if subjects who were non-users were familiar with the brand prior to the trial (something which would be crucial to any explanation). Branthwaite and Cooper continue:

Taking branded tablets, which people believe from past experience, hearsay, advertising, etc., are likely to relieve their headache, may divert attention from the headache or its sources, encourage a sense of wellbeing and coping, and therefore accelerate recovery. . . . Nevertheless, the branding was effective for non-users as well as users, which may suggest that the particular brand tested has a general reputation for efficacy. (1578, emphasis added)

Again, this is all based on what patients might have grounds for believing. And while the authors do discuss briefly the possibility that brand reputation might play a role, they do not discuss what might constitute that reputation, much less how "reputation" is itself not reducible to a set of beliefs. Our thought is that these oversights emerge because the authors operate exclusively within the paradigm of expectation. The authors are looking for something in the brand that can 
be captured in the form of beliefs that might, in turn, constitute the expectation for those receiving the pills; in doing so, the authors overlook the role of meaning that does not take that form, the sort of meaning that might emerge from associations the brand has, from connotations of the brand name, including the font, color, and other packaging design elements.

We can look at this from another perspective. While we might consider it likely that the subjects believe that aspirin will relieve their headache, it is, we propose, less likely that the same subjects believe that aspirin with the same composition, only packaged and named differently, will be more likely to relieve their headache or relieve it more quickly. Why would they believe this? Nevertheless, the meaning the branded aspirin has for them, in contrast to the non-branded one, will vary, owing to conative and associative factors. We do not seek to pursue this point further here, rather we seek to make a plea for trials incorporating social researchers so that we might obtain the sort of data that enables better understanding. RCTs alone, without incorporating social researchers, leave too much to speculation. ${ }^{2}$

So, while in the case of the coffee example, the response-expectancy explanation seems viable, because the example is one where belief seems to readily play an explanatory role, does it seem viable in the case of pill color or branding as drivers of the placebo response? We suggest not.

What of expectancy now? If we hold on to expectancy as an explanation for placebo responses, then we need to provide warrant for our having extended the grammar of "to expect," such that it no longer entails an intentional object for the expectation, which gives the expectation its content. A modification of this kind would mean access to the relevant epistemic resources would no longer be an issue, which is important because-recall - they are simply unavailable in many cases of placebo responses. However, such a move would amount to a significant extension to the grammar of "to expect" and its use, and would therefore find no support in current meaningful uses of the term.

We can put the argument of the last paragraph less abstractly: if you want to stick with expectancy, you owe us an explanation as to how someone can expect something while not being in a position to know or specify what it is they are expecting - in other words, how can one expect something or a state of affairs without being able to specify or represent the something or state of affairs that they are expecting? One might be tempted here to say, "Well, one can be in a state of expectancy, which is nonspecific or non-object-directed," and we can concede that this is possible, though we suggest this would be better described as anticipation. However, such nonspecific, or contentless, expectancy (or anticipation) is not what is being appealed to in the response-expectancy explanation.

${ }^{2}$ As will become clearer in the final section of this paper, we propose incorporating ethnomethodologically informed researchers in trials. 
Nonspecific expectancy cannot be marshalled as a cause for a particular placebo response, because the response-expectancy explanation relies on the specific content of the expectation or expectancy being the very thing that generates the response. Put another way, for those who would defend the response-expectancy explanation, it is not merely being in a state of expectation that explains a placebo response, but the content of the expectation or expectancy. It is the object of the expectation - that which is expected - that does the lifting.

\section{The Meaning Response}

We might, therefore, go another way. We could leave the grammar of "to expect" as it is, stop trying to square this particular circle, and instead drop the overly restrictive reliance on response-expectancy as a global explanation for placebo responses. Then, as an alternative, we might talk more broadly about meaning responses, and set about, via ethnographic investigations of people responding in medically significant ways to pharmacologically and surgically inert interventions, the task of gaining a better understanding of medically significant meaning responses. Indeed, if we do so, we can forgo the preoccupation with the somewhat misleading term placebo, because medically significant meaning responses are present whether what is being administered is a dummy pill or a pharmacologically active pill, whether we are studying sham or dummy surgical interventions, or whether we are studying surgery. What has been referred to as the "placebo effect" or "placebo response" is but one manifestation of the meaning response, the manifestation that involves sham treatment. To conclude that what is significant or important is the nature of the sham part of the treatment is to miss what is genuinely worthy of investigation here: the role of meaning in medical practice and health.

We are not talking about the world having meaning in a way that demands we first have the resources to form beliefs about (possible) states of affairs in the world, for we don't need to expect something to have a positive effect on our health us for us to be undergo a significant improvement in health when we have received a sham treatment. Rather, we move beyond such a representationalist picture of cognition, to more ecological (Costall 1995; Gibson 1986; Heft 1989), enactive (Ongaro and Ward 2017) or even ethnomethodologically informed accounts (Garfinkel 1967).

How do we advance from this point? The two candidate explanations we have rejected share three things in common. First, both seek to provide a quasi-mechanistic explanation for placebo responses. In the tradition of a certain understanding of what counts as a scientific explanation, these two candidate explanations seek to provide a general explanation that will cover all particular instances. Second, they both reject any appeal to culture. They forgo any attempt to explain those people who respond to placebos in a way that might acknowledge and then unpack the role of enculturation in the constitution of the person-as-member-of- 
a-social-order who responds in a medically significant way to the administration of a sham intervention. Instead, they focus on behavioral conditioning (stimulus-response explanations), innate capacities, or learning (response-expectancy explanations). Finally, both explanations, in taking placebo responses as their subject, run the risk of allowing their investigations to be framed and constrained by the administration of dummy pills and sham surgery and seek to explain this, when this is just one small part of the wider phenomenon of the medically significant role the meaning of social practices, social settings, packaging design, and branding play in medical treatment. The ways in which human beings, as members of social orders, come to meet, engage in, and even modify their world cannot be reduced to innate characteristics, conditioning, and learning. That which we gesture at when we talk of culture and enculturation needs to be included and explicated.

In the case of placebos, on the one hand we know that the intended "intervention" is sham-the pill is a dummy pill, and the surgical intervention is a mocked-up "intervention" - and this gives us one condition for something being a placebo. On the other hand, we have seen that explaining what is generating the response here via appeals to conditioning or expectation doesn't work. The explanation is not that people are responding because they are conditioned to do so, in a manner that depicts those people as akin to functional artefacts, such as smoke detectors (where the conditioning of the person plays the role of the design of the artefact). Nor is it to be explained in all cases by claiming people are responding to their expectations, in a manner that depicts those people as data-processing devices. Instead, we propose that what has been referred to as the "placebo response" is actually a type of meaning response. Moreover, when we talk of meaning response, we are cognizant that responders are human beings who are members of a society, and who have gone through processes of enculturation. People respond to the meaning that complex social practices have for them, where those practices involve them as co-participants and co-producers of the order one finds therein, and they do so by virtue of their enculturation.

Where does this leave us? Response-expectancy is an explanation that invokes representationalist accounts of cognition, but which fails in its own terms. Our criticism shows that response-expectancy demands propositional content that is often simply unavailable. Moreover, our criticism goes deeper, questioning the representationalist paradigm within which response-expectancy operates. Recently, non-representationalist approaches to cognition have reemerged or risen to prominence. In seeking to explain the medically significant meaning response, we propose one needs to look to these non-representationalist alternatives, especially radical enactivism (Hutto and Myin 2013, 2017), ecological psychology (Chemero 2009; Costall 1995; Gibson 1986; Heft 1988), and our favored approach, ethnomethodology (Coulter 1991; Garfinkel 1967). We will discuss the ethnomethodological approach in the penultimate section of this paper. 


\section{Losing THE PHENOMENON}

Being in thrall to a specific form of explanation. . .

We propose that the first two of our candidate explanations of placebo responses, conditioning and expectancy, result in the loss of the phenomenon.

The first two, related, reasons for this, we suggest, are because (1) there is a background assumption that only certain forms of explanation are scientifically respectable, if one is to meet the naturalistic demand; and (2) the (already mentioned) commitment to representationalist conceptions of cognition set-off investigations on dead-end routes. These assumptions and commitments impose constraints on candidate explanations because they serve to a priori restrict what is to count as an acceptable explanation. Non-adhering forms of explanation, which reject those assumptions and commitments, are thus treated as "scientifically" disreputable or as failing to meet the representationalist demand that cognition is necessarily representational.

These constraints can serve to prevent researchers from looking beyond innate characteristics of the species, behavioral conditioning, and proactive learning. We should like to suggest that it is exactly what falls outside the space of innate characteristics, behavioral conditioning, and proactive learning that is central to understanding placebo and meaning responses. We will say more about this in the next section, but what we're interested in is what generally goes by the name of "culture": the norms, rules, and situated forms of practice that give order to social settings and render them intelligible or accountable-in other words, for which it is possible to produce accounts - as social settings (as opposed to their being reducible to concatenations of random acts of individuals).

In addition to the "unrefined naturalist" and the "representationalist" constraints, we suggest that there is another problem, a problem that arises from misidentifying the true phenomenon in need of explanation. When investigating the phenomenon of people responding in medically significant ways to dummy pills or sham surgery, one can quickly become drawn into considerations of a much larger domain of phenomena that are significant for understanding medical practice more broadly. To allow one's focus to remain fixed on the administering of dummy pills and sham surgery is akin to trying to understand a duck by focusing only on that part of the duck that is above water. For sure, the placebo controls in RCTs allow us to isolate and therefore throw into sharp relief the meaning response, by allowing us to control for it and differentiate medically significant meaning responses to the administration of dummy pills or sham surgery from regression to the mean, Hawthorne effects, natural history effects, and so on. However, we should not confuse our ability to isolate the meaning response in this way with the nature of the phenomenon: there is much more to the phenomenon than responses to dummy pills and sham surgery. In order to understand the 
nature of the meaning response, we will need to broaden our investigation and methods beyond RCTs, fMRI scans, and theories of expectation/expectancy.

\section{Finding The PHENOMENON}

EM fieldwork: social practices, members, and the co-production of social settings

As our argument has unfolded, we have gestured at enculturation and culture as relevant to any adequate understanding of the meaning response and how it figures in producing what have historically been referred to as "placebo responses." It might be tempting, therefore, to invoke culture-sets of social norms, rules, and practices - as an explanatorily primitive category and perhaps propose a "culture model" or "meaning model." However, learning from the ethnomethodologists, we recommend resisting such a temptation (Button 1991; Garfinkel 1967; Garfinkel and Rawls 2002; Heritage 1991). For, as Harold Garfinkel has demonstrated, culture is not the primitive explanation for the orderliness of social action, but rather the (co-)product of social action, which is engaged in by members of a society who have gone through processes of enculturation: learning a language, exposure to narratives, and learning to see patterns, order, and significance in behavior, symbols, and institutions. Garfinkel's insights emerged from studies in which he, his colleagues, and his students created scenarios in which they might observe social action in situations in which shared cultural norms, rules, and familiar forms of conduct were absent or destabilized. What is observed in such cases is that co-participants create, repair, and maintain social order and the social setting in situ, by drawing on endogenous - in other words, internally produced or self-furnishing-resources: they draw on their enculturation in situ and do so in a methodic manner that serves to frame the purposive or intentional actions undertaken in the same scenarios. Consider, for example, a conversation. The parties to a conversation make intentional or purposive contributions to that conversation, but the background against which they do so-the conditions that make this a conversational setting in which they can make those purposive contributions - is also generated, maintained, and, if required, repaired by those same participants (as members of the social order) through their methodic actions. What goes for conversation, goes for drug administering, clinical encounters such as a visit to the physician, and so on. These settings involve purposive and intentional action undertaken against a background, or in a setting, furnished through the methodic action of the participants.

Observing the role of methodic action helps us see that invoking culture as the primitive explanation or condition for social order, or the existence of social facts, is to misrepresent matters. Ethnomethodological studies show that culture is better understood as the product of members' methodic actions and interactions, which provide the framework in which purposive or intentional action and in- 
teraction can take place. ${ }^{3}$ Individuals are members of social orders, having passed through processes of enculturation, or as Aristotle would have put it, having been endowed with a second nature (in addition to their purely biological nature).

Let us be clear about what we are not arguing here. We are not arguing that there is no culture or process of enculturation, and that there are only individuals and their spontaneously produced actions. The point is rather that explanatory appeals to culture (or norms, social structure, or social facts) are not appeals to some primitive ontological entity or entities that are constitutively independent of the action, co-action, and interaction of members of social orders, but which serve as a condition for the order we find in social settings. Rather, the point is that the "order" in "social order" is to be found by observing the methodic actions of members of that social order in their interactions with each other and by representing these actions in concepts available to the members. This endogenously produced order is what makes social settings more than merely the sum or concatenation of spontaneously produced, ahistorical, and acultural individual acts. Culture is seen through, observed in, and lives as the methods used by members of social orders in social settings extended over time.

That which is usually referred to as "culture" is not reducible to the products of learning, nor is it reducible to conditioning. There is something else. Some of this comes from becoming proficient in language(s), the sorts of conceptual horizons that are established, and the cognitive penetration enabled by doing so, and how this is integrated into the shared practices that anthropologists and ethnographers study. Some of it comes from acting in and on the world, and how this shapes us, as studied by enactivists. And some of this comes from institutional constraints and enabling conditions. This is what we gesture at when we talk of "enculturation." Moreover, we gain access to these things and we demonstrate their presence as constraints or as enabling conditions (social norms and rules), not through social theorizing, but through observing members of societies co-producing, repairing, maintaining, and challenging these constraints and enabling conditions in their interactions.

This insight into the "place" of culture and the role of ethno-methods in its forming provides us with guidance in the work we must do if we are to understand the relationship between meaning and culture. If, as we are proposing and as the data suggests, the meaning a social practice has for a subject can generate medically significant responses, then we need to do more than simply appeal to culture to explain this. Culture is produced, repaired, and maintained, formed and reformed, and is observable through the methodic meaningful actions and interactions of members of society.

${ }^{3}$ There are illuminating parallels here with Wittgenstein's remarks at $\$ \$ 241-242$ of his Philosophical Investigations. This has been fruitfully explored in writings over a period of 40 years by the "Manchester Ethnomethodologists," including Wes Sharrock and Jeff Coulter, along with various coauthors. 
For example, the logic of - or the order one finds in - traffic flow is produced by the methodic action and interaction of drivers, but it is not reducible to their intentional actions, any more than it is to traffic laws and road signs. The logic of institutions is produced by the methodic action and interaction of the members who inhabit them- how they enact the rules and regulations, how they inherit, understand, and enact the purpose of the institution and negotiate the interplay between this and their own purposes, and so on-but it is not reducible to nor explained by the intentional actions of members, nor by the institutional rules and regulations.

What we propose is this: the logic of the meaning response- the way in which people respond in medically significant ways to meaning-is, we suggest, co-produced by the (non-intentional) methodic actions of participants in medical settings, including drug trials. It cannot be reduced to the intentional actions and expectations of those same participants.

To conclude this section, we might put things in the following way: those who are responsive in medically significant ways to the meaning found in social settings such as the clinical encounter (in which drugs are administered, or surgery is conducted) and are so in ways that cannot be reduced to a response to the pharmacology of the drug or to the cuts of the surgeon's scalpel, are also enculturated members of society. As such, they both inherit that society's cultural traditions and draw upon them as they interact with others, in a way that results in the co-production, maintenance, and repair of the order (or logic) that is both constitutive of the social setting of which they are part and that renders that setting as something for which we can provide an account. What it also does is serve to potentially differentiate that clinical social setting from other clinical social settings that are in other ways ostensibly the same: how we identify the cultural differences. What we propose is required, if we are to make progress in understanding meaning responses (and therefore understand what has historically been referred to as the placebo response), is the conducting of ethnomethodological fieldwork in placebo controls in medical trials. We currently have many neuroscientific studies of placebo controls, based on producing and analyzing AMRI scans. What we propose is to add ethnomethodological fieldwork studies, so that we might start to build a picture of the logic of meaning responses.

\section{CONCLUDING THOUGHTS}

In summary, pace Hróbjartsson and Gøtzsche and those persuaded by them, there is a phenomenon here in need of explanation. A significant percentage of people respond in medically significant ways, under certain conditions, and these responses cannot be explained away as mislabelled natural history effects, regression to the mean, or the Hawthorne effect. 
The candidate explanations for this phenomenon that appeal to classical conditioning and expectancy face significant difficulties. Were one inclined to operate with a Lakatosian framework, one would, we suggest, be forced to declare both the stimulus-response and response-expectancy research programs degenerative, and terminally so. Another research program is required, and we propose the meaning response provides the framework that might best guide that program.

The spontaneous philosophy that we argue underpins the appeal of both stimulus-response and response-expectancy explanations is motivated by an unrefined naturalism, in which explanations are accepted as meeting the naturalistic demand only inasmuch as they ape explanations found in currently well-established natural science disciplines. We instead work with an understanding of the adequacy of explanation that is domain-specific and builds up from an understanding of the nature of the phenomena. Unrefined naturalism leads to a loss of the phenomenon, and in the present context, it leads one to mistake a token instance of the phenomenon (the human capacity to respond in medically significant ways when pharmacologically inert pills and sham surgery are administered) with the phenomenon itself (the human capacity to respond in medically significant ways to the meaning certain social practices have for them).

We therefore propose that ethnomethodologically informed fieldwork be incorporated in order to direct us to the endogenous methods employed by members of societies in the co-production of the order and meaning of clinical settings, thereby exhibiting the role meaning has in the efficacy of medical practice. In this way, significant ground can be made in understanding the phenomenon currently referred to as "placebo."

\section{REFERENCES}

Ader, R. 1988. "The Placebo Effect as Conditioned Response.” In Experimental Foundations of Behavioral Medicine: Conditioning Approaches, ed. R. Ader, H. Weiner, and A. Baum, 47-66. London: Psychology Press.

Blease, C. R., F. L. Bishop, and T. J. Kaptchuk. 2017. "Informed Consent and Clinical Trials: Where Is the Placebo Effect?” BMJ 356: j463. DOI: 10.1136/BMJ.J463.

Branthwaite, A., and P. Cooper. 1981. "Analgesic Effects of Branding in Treatment of Headaches." BMJ 282 (6276): 1576-78. DOI: 10.1136/BMJ.282.6276.1576.

Button, G. 1991. Ethnomethodology and the Human Sciences. Cambridge: Cambridge University Press.

Cattaneo, A. D., P. E. Lucchelli, and G. Filippucci. 1970. "Sedative Effects of Placebo Treatment." Eur J Clin Pharmacol 3 (1): 43-45. DOI: 10.1007/BF00560290.

Chemero, A. 2009. Radical Embodied Cognitive Science. Cambridge: MIT Press.

Costall, A. 1995. "Socializing Affordances." Theor Psychol 5 (4): 467-81. DOI: 10.1177/0959354395054001.

Coulter, J. 1991. "Cognition: Cognition in an Ethnomethodological Mode." In Ethnomethodology and the Human Sciences, ed. G. Button, 176-95. Cambridge: Cambridge University Press. 
Garfinkel, H. 1967. Studies in Ethnomethodology. Englewood Cliffs: Prentice Hall.

Garfinkel, H., and A. W. Rawls. 2002. Ethnomethodology's Program: Working Out Durkheim's Aphorism. New York: Rowman and Littlefield.

Gibson, J. J. 1986. The Ecological Approach to Visual Perception. London: Psychology Press, 2014.

Heft, H. 1988. "The Development of Gibson"s Ecological Approach to Perception." J Environ Psychol 8 (4): 325-34. DOI: 10.1016/S0272-4944(88)80038-0.

Heft, H. 1989. "Affordances and the Body: An Intentional Analysis of Gibson Ecological Approach to Visual-Perception." J Theor Soc Behav 19 (1): 1-30. DOI: 10.1111/ j.1468-5914.1989.tb00133.x.

Heritage, J. 1991. Garfinkel and Ethnomethodology. Oxford: Polity Press.

Howick, J. 2016. "What"s the Truth About Placebo Effects?" Huffington Post, July 15. http://www.huffingtonpost.co.uk/jeremy-howick/placebo-effects_b_10906256. html.

Howick, J., et al. 2013. "Are Treatments More Effective Than Placebos? A Systematic Review and Meta-Analysis." PLoS One 8 (5): 1-8. DOI: 10.1371/journal.pone.0062599.

Hróbjartsson, A., and P. C. Gøtzsche. 2001. "Is the Placebo Powerless?" N Engl J Med 344 (21): 1594-1602. DOI: 10.1056/NEJM200105243442106.

Hróbjartsson, A., and P. C. Gøtzsche. 2010. "Placebo Interventions for All Clinical Conditions (Review)." Cochrane Database Syst Rev 1: 1-3. DOI: 10.1002/14651858. CD003974.pub3.Copyright.

Hutto, D. D., and E. Myin. 2013. Radicalizing Enactivism: Basic Minds Without Content. Cambridge: MIT Press.

Hutto, D. D., and E. Myin. 2017. Evolving Enactivism: Basic Minds Meet Content. Cambridge: MIT Press.

Kirsch, I. 1985. "Response Expectancy as a Determinant of Experience and Behavior." Am Psychol 40 (11): 1189-1202. DOI: 10.1037/0003-066X.40.11.1189.

Kirsch, I. 1997. "Response Expectancy Theory and Application: A Decennial Review." Appl Prevent Psychol 6 (2): 69-79. DOI: 10.1016/S0962-1849(05)80012-5.

Kirsch, I., and L. J. Weixel. 1988. "Double-Blind versus Deceptive Administration of a Placebo." Behav Neurosci 102 (2): 319-23. DOI: 10.1037/0735-7044.102.2.319.

Lucchelli, P. E., A. D. Cattaneo, and J. Zattoni. 1978. "Effect of Capsule Colour and Order of Administration of Hypnotic Treatments." Eur J Clin Pharmacol 13 (2): 153-55. DOI: 10.1007/BF00609760.

Moerman, D. E. 2000. "Cultural Variations in the Placebo Effect: Ulcers, Anxiety, and Blood Pressure." Med Anthropol Q 14 (1): 51-72.

Moerman, D. E. 2002. Meaning, Medicine, and the "Placebo Effect." Cambridge: Cambridge University Press.

Ongaro, G., and D. Ward. 2017. "An Enactive Account of Placebo Effects." Biol Philos 32 (4): 507-33. DOI: 10.1007/s10539-017-9572-4.

Rescorla, R. A. 1988. "Pavlovian Conditioning: It's Not What You Think It Is." Am Psychol 43 (3): 151-60. DOI: 10.1037/0003-066X.43.3.151.

Wittgenstein, L. 2009. Philosophical Investigations. 4th ed. Oxford: Blackwell. 